\title{
Application of fluorine-based threshold activation detector for neutron flux calculation from D-T neutron generator
}

\author{
Paweł Sibczyński*, Andrzej Brosławski*, Szymon Burakowski*, Arkadiusz Chłopik*, Marek Dryll*, \\ Andrzej Dziedzic*, Michał Gierlik*, Krystian Grodzicki*, Łukasz Kaźmierczak*, Tomasz Krakowski*, \\ Kamil Niechcielski*, Jacek Rzadkiewicz*, Ivan Sliphukin*, Krystian Trela*, Arkadiusz Urban*, \\ Kacper Zezuliński*, Alessandro Iovene ${ }^{\dagger}$ and Carlo Tintori ${ }^{\dagger}$ \\ *National Centre for Nuclear Research (NCBJ), A. Soltana 7, 05-400 Otwock, Poland \\ Electronics and Detection Systems Division (TJ2) \\ Email: pawel.sibczynski@ncbj.gov.pl \\ $\dagger$ CAEN S.p.A., Via Vetraia 11, 55049 Viareggio (LU), Italy
}

\begin{abstract}
In this paper we propose a method of fast neutron flux estimation from a pulsed D-T neutron generator with application of single $\mathbf{C a F}_{2}$ scintillation crystal. The analysis method relies on ${ }^{19} \mathbf{F}(n, \alpha){ }^{16} \mathbf{N}$ threshold activation reaction having neutron energy threshold at $1.6 \mathrm{MeV}$. As a result, the ${ }^{16} \mathrm{~N}$ undergo $\beta^{-}$decay with half-life of $7.1 \mathrm{~s}$, emitting $\beta$ particles with endpoint up to $10.4 \mathrm{MeV}$ in the scintillator medium. Integration of the $\beta$ distribution curve, preceded by calculation of $(n, \alpha)$ rate on $F$ with Monte Carlo N-Particle Transport Code v6 (MCNP6) for fixed geometry, allows to estimate the neutron flux in $4 \pi$ per second within few minutes.
\end{abstract}

Index Terms-neutron detection, Threshold Activation Detection, scintillator, neutron generator

\section{INTRODUCTION}

One of a technique useful for fast neutron detection is known as a Threshold Activation Detection (TAD) [1], [2]. It relies on registration of characteristic radiation emitted from specific nuclei after threshold reaction with neutrons. A possible approach is to use a scintillation material containing itself a material, which undergoes the activation reaction, such as fluorine. In this work we investigated a $\mathrm{CaF}_{2}$ scintillator, which mass fraction of $\mathrm{Ca}$ and $\mathrm{F}$ is about $50 / 50 \%$ [3]. The F nuclei, present in the scintillator medium, undergo i.e. ${ }^{19} \mathrm{~F}(n, \alpha){ }^{16} \mathrm{~N}$ reaction, resulting in emission of high energy beta particles (endpoints at $10.4 \mathrm{MeV}$ and $4.3 \mathrm{MeV})$ and $\gamma$ rays $(6.1 \mathrm{MeV})$ with the half-life of $7.1 \mathrm{~s}$. The $(n, \alpha)$ reaction cross section reaches about $100 \mathrm{mb}$ at about $5 \mathrm{MeV}$ (see Fig. 1), which is sufficient for such application. In this work, we propose a new method of neutron flux estimation from deuteriumtritium (D-T) neutron generators operated in pulse mode. An undoped $\mathrm{CaF}_{2}$ with size of $\varnothing 5$ " $\times 3$ " was used, however, other scintillators containing fluorine such as EJ-313/BC-509, $\mathrm{BaF}_{2}$ or fluorocarbon plastic [4] can be also considered for that application. The method usefulness for

This project has received funding from the European Union's Horizon 2020 research and innovation programme under grant agreement No 653323 and CentriX project No RPMA.01.01.00-14-8516/17.

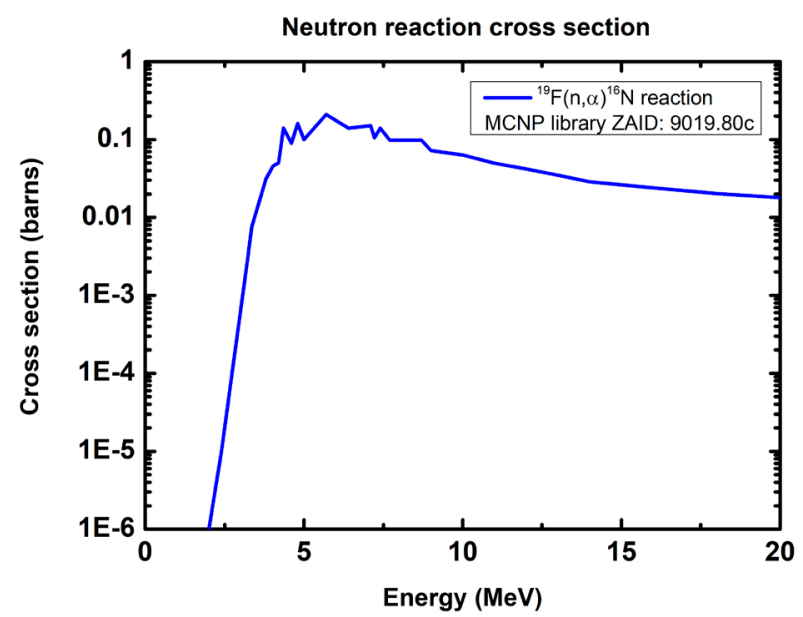

Fig. 1. The ${ }^{19} \mathrm{~F}(n, \alpha)^{16} \mathrm{~N}$ neutron cross section used in the MCNP simulations, a ZAID 9019.80 c library was chosen.

deuterium-deuterium (D-D) neutron generators flux monitoring will be also discussed. The $\mathrm{CaF}_{2}$ scintillator was previously used for simultaneous detection of prompt photofission neutrons and delayed $\gamma$-rays from nuclear materials, potentially hidden in cargo container. These field trials were performed at Maasvlakte Rotterdam Seaport in the frame of C-BORD project.

\section{Methodology}

\section{A. Experimental setup}

Fig. 2 presents the experimental setup at NCBJ measurement facility, equipped with a Sodern Genie 16D D-T neutron generator. Data were registered with a CAEN DT5730 8 channel $500 \mathrm{MS} / \mathrm{s}$ desktop digitizer with DPP-PSD firmware and DigiTes 4.5.10 control software. The $1 \mathrm{~ms}$ excitation and $10 \mathrm{~ms}$ repetition neutron generator pulse mode was set. The trigger width was shaped by a laboratory-grade digital logic pulse shaper based on Arduino, 


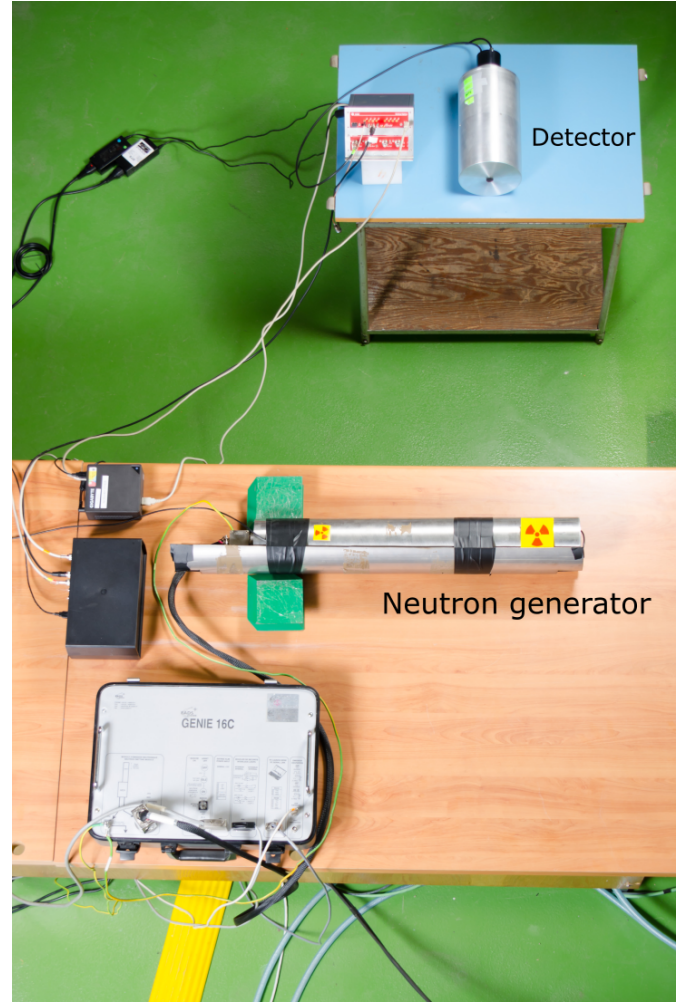

Fig. 2. The experimental setup for measurements of a neutron generator flux.

which blocked acquisition for $5 \mathrm{~ms}$ after each pulse in order to prevent detection of neutron capture $\gamma$-rays. The detector was placed $1 \mathrm{~m}$ from neutron generator tritium target and $83 \mathrm{~cm}$ above the concrete floor level. Each acquisition run last 10 minutes.

\section{B. Monte Carlo simulations and data analysis}

An MCNP v6.11 software package [5], [6] was used for investigation of the $\mathrm{CaF}_{2}(n, \alpha)$ reaction rate, expressed per one source neutron. We used average flux tally with FM4 card in order to calculate the reaction rate separately for $\mathrm{Ca}$ and $\mathrm{F}$ and run $1 \mathrm{E} 8$ histories. The obtained $(n, \alpha)$ rates for D-T and D-D neutron generators were presented in Tab. I. The uncertainties (expressed in percentage of the expected value) were taken from the MCNP calculation output, which are related only with the number of launched histories. The MCNP geometry model is shown in Fig. 3.

TABLE I

The Simulated $(n, \alpha)$ REACTION RATES IN THE $\mathrm{CAF}_{2}$ Detector.

\begin{tabular}{|c|c|}
\hline Neutron generator & $(n, \alpha)$ rate for ${ }^{19} \mathbf{F}$ \\
\hline D-T $\left(E_{n}=14 \mathrm{MeV}\right)$ & $1.37 \times 10^{-5} \pm 0.0041 \%$ \\
$\mathrm{D}-\mathrm{D}\left(E_{n}=2.5 \mathrm{MeV}\right)$ & $6.46 \times 10^{-9} \pm 0.0037 \%$ \\
\hline
\end{tabular}

The $(n, \alpha)$ reaction rate for the simulated setup for D-T neutron generator is over four orders of magnitude greater than that for D-D one. This is in line with expectations, as the total $(n, \alpha)$ cross section for the $2.5 \mathrm{MeV}$ neutrons is significantly

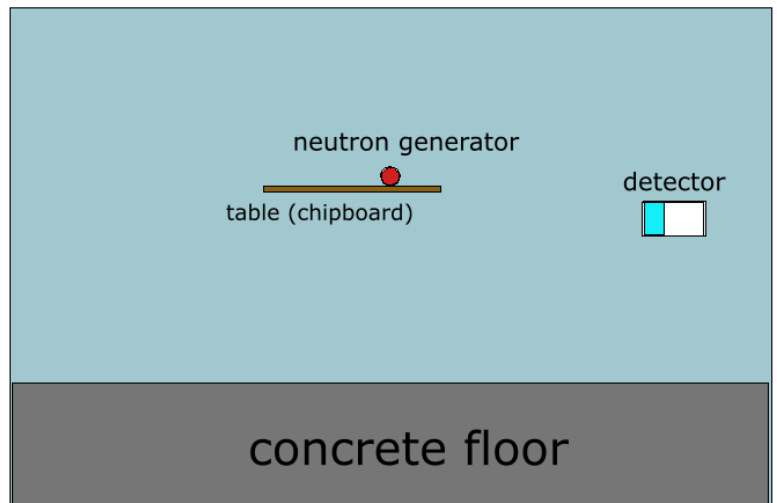

Fig. 3. Visualization of the MCNP geometry model.

lower than that for the D-T neutron generator. A D-D neutron generator would need to provide neutron flux of $2.5 \times 10^{11} \mathrm{n} / \mathrm{s}$ to obtain the same statistics as the Sodern D-T generator at $90 \mathrm{kV} / 40 \mu \mathrm{A}$.

After the $(n, \alpha)$ reaction rate calculation, one need to calculate the number of generated $\beta$ particle with the endpoint at $10.4 \mathrm{MeV}$. The intensity of this transition is $28 \%$. In contrast, the intensity of the $4.3 \mathrm{MeV}$ transition is $66 \%$. The exemplary energy spectrum for neutron generator $\mathrm{HV}$ of $90 \mathrm{kV}$ and current of $40 \mu \mathrm{A}$ is presented in Fig. 4.

The $\beta$ continuum with endpoints at $4.3 \mathrm{MeV}$ and $10.4 \mathrm{MeV}$ as well as $6.1 \mathrm{MeV} \gamma$-rays are clearly visible. The $\beta$ continuum energy distribution was fitted with use of the following approximation (1):

$$
\mathrm{N}(\mathrm{E}) \sim \mathrm{C} / \mathrm{c}^{5} \times \sqrt{\left(\mathrm{E}^{2}+2 \mathrm{Em}_{\mathrm{e}} \mathrm{c}^{2}\right)} \times(\mathrm{Q}-\mathrm{E})^{2} \times\left(\mathrm{E}+\mathrm{m}_{\mathrm{e}} \mathrm{c}^{2}\right),
$$

where $Q$ - beta endpoint, $E$ - electron energy, $m_{e}$ - electron mass, $c$ - speed of light, $C$ - constant.

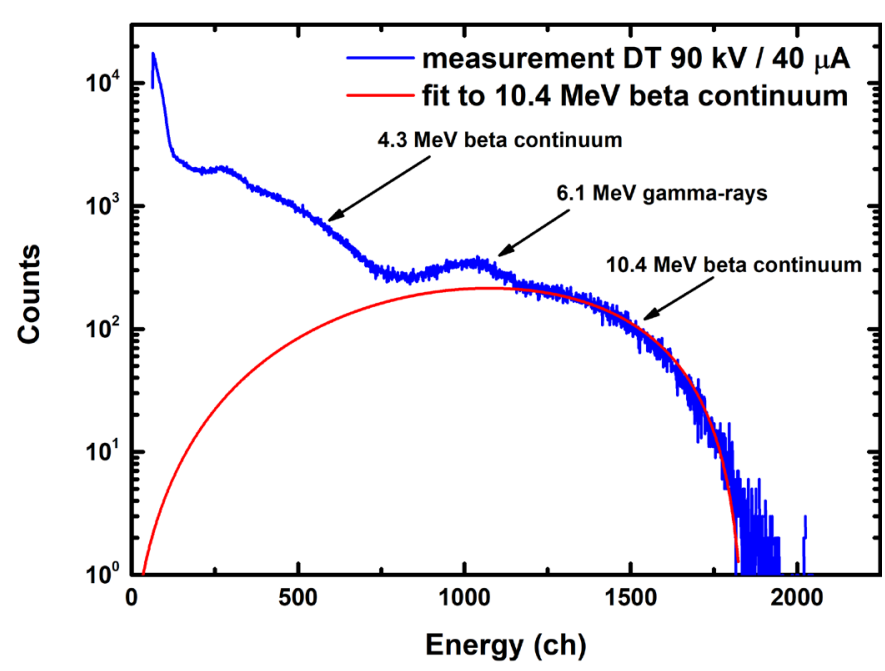

Fig. 4. The $\mathrm{CaF}_{2}$ energy spectra after $600 \mathrm{~s}$ of exposition to $14 \mathrm{MeV}$ neutrons from $\mathrm{D}-\mathrm{T}$ neutron generator. 


\section{Results}

The calculated neutron flux from the D-T neutron generator is presented in Fig. 5. The neutron flux per second was calculated with the following equation (2):

$$
\phi_{n}=\frac{\int_{0}^{Q} N(E) d E}{R(n, \alpha) \times B},
$$

where $\int_{0}^{Q} N(E) d E$ is the integral over the beta energy spectrum distribution from 0 to the beta endpoint $\mathrm{Q}, R(n, \alpha)$ is the $(n, \alpha)$ reaction rate on ${ }^{19} \mathrm{~F}$, obtained from the MCNP simulations, and $\mathrm{B}$ is the branching ratio of $(n, \alpha)$ for 10.4 $\mathrm{MeV}$ beta endpoint, equal to 0.28 . Results were normalized to obtain the neutron flux per one second. The obtained results are in very good agreement with the neutron flux data provided by the neutron generator manufacturer.

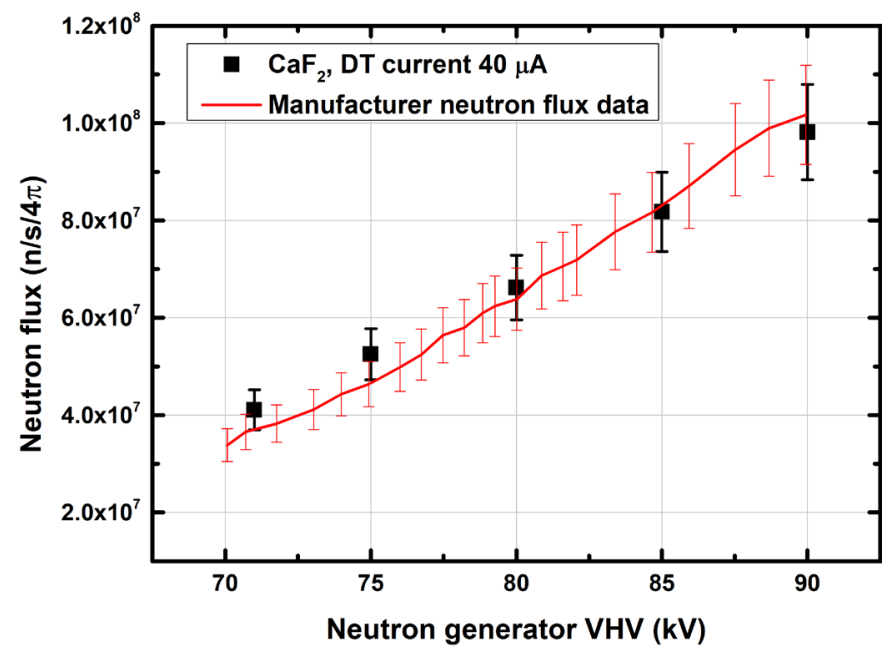

Fig. 5. Calculated neutron flux from Sodern Genie 16D neutron generator with use of $\mathrm{CaF}_{2}$ detector and comparison with the manufacturer data.

\section{CONCLUSIONS}

We presented the approach for fast neutron flux estimation from D-T neutron generator with use of a single $\mathrm{CaF}_{2}$ as the threshold activation detector. Main advantage of the method is the fact that neither activation foils nor High Purity Germanium (HPGe) detector is required to perform the flux calculation. After preceded MCNP simulations, required for $(n, \alpha)$ rate estimation at fixed detector and neutron generator positions, the neutron flux can be calculated in real time even for intense and short neutron pulses. This solution can be exceptionally useful in neutron generator test-bench facility, where at fixed positions one need to calculate the absolute neutron flux for any type of D-T neutron generator.

\section{REFERENCES}

[1] T. Gozani, M. J. King, and J. Stevenson, "The investigation of fast neutron Threshold Activation Detectors (TAD)," J. Inst., vol. 7, pp. C02042C02042, Feb. 2012.
[2] J. Stevenson, T. Gozani, M. Elsalim, C. Condron, and C. Brown, "Linac based photofission inspection system employing novel detection concepts," Nuclear Instruments and Methods in Physics Research Section A: Accelerators, Spectrometers, Detectors and Associated Equipment, vol. 652, pp. 124-128, Oct. 2011.

[3] P. Sibczynski, A. Dziedzic, K. Grodzicki, J. Iwanowska-Hanke, M. Moszyński, L. Swiderski, A. Syntfeld-Każuch, D. Wolski, F. Carrel, A. Grabowski, M. Hamel, F. Laine, A. Sari, A. Iovene, C. Tintori, C. Fontana, and F. Pino, "New perspectives for undoped $\mathrm{CaF}_{2}$ scintillator as a threshold activation neutron detector," EPJ Web of Conferences, vol. 170, p. 07012, 2018.

[4] M. Hamel, P. Sibczynski, P. Blanc, J. Iwanowska, F. Carrel, A. SyntfeldKażuch, and S. Normand, "A fluorocarbon plastic scintillator for neutron detection: Proof of concept," Nuclear Instruments and Methods in Physics Research Section A: Accelerators, Spectrometers, Detectors and Associated Equipment, vol. 768, pp. 26-31, Dec. 2014.

[5] T. Goorley, M. James, T. Booth, F. Brown, J. Bull, L. J. Cox, J. Durkee, J. Elson, M. Fensin, R. A. Forster, J. Hendricks, H. G. Hughes, R. Johns, B. Kiedrowski, R. Martz, S. Mashnik, G. McKinney, D. Pelowitz, R. Prael, J. Sweezy, L. Waters, T. Wilcox, and T. Zukaitis, "Initial MCNP6 Release Overview," Nuclear Technology, vol. 180, pp. 298-315, Dec. 2012.

[6] J. K. Shultis and R. E. Faw, "An MCNP primer," tech. rep., Dept. of Mechanical and Nuclear Engineering, Kansas State University, Manhattan, KS 66506, 2011. 